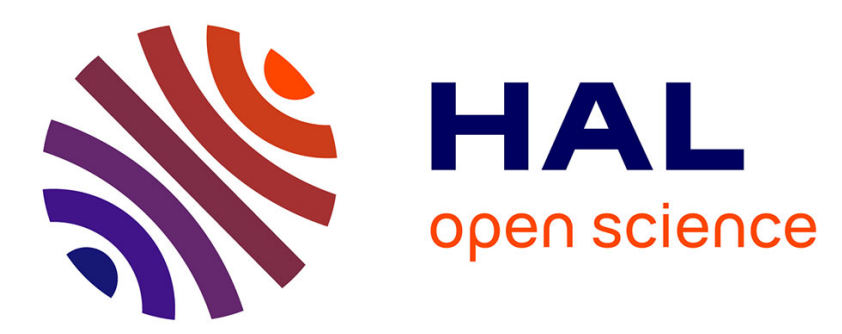

\title{
Témoins manuscrits connus et inconnus du Rapport de Pilate à Tibère (CANT 65 et 66)
}

Anne-Catherine Baudoin

\section{To cite this version:}

Anne-Catherine Baudoin. Témoins manuscrits connus et inconnus du Rapport de Pilate à Tibère (CANT 65 et 66). Revue d'études augustiniennes et patristiques, 2008, 54, pp.183-206. halshs01293241

\section{HAL Id: halshs-01293241 \\ https://shs.hal.science/halshs-01293241}

Submitted on 25 Mar 2016

HAL is a multi-disciplinary open access archive for the deposit and dissemination of scientific research documents, whether they are published or not. The documents may come from teaching and research institutions in France or abroad, or from public or private research centers.
L'archive ouverte pluridisciplinaire HAL, est destinée au dépôt et à la diffusion de documents scientifiques de niveau recherche, publiés ou non, émanant des établissements d'enseignement et de recherche français ou étrangers, des laboratoires publics ou privés. 
Revue d'études augustiniennes et patristiques, 54 (2008), 183-206.

\section{Témoins manuscrits connus et inconnus du Rapport de Pilate à Tibère (CANT 65 et 66)}

Le Rapport de Pilate à Tibère est un texte apocryphe chrétien écrit à la première personne; le narrateur, Pilate, s'adresse à l'empereur Tibère pour lui raconter les événements qui se sont déroulés peu de temps auparavant à Jérusalem. Il explique qui est ce Jésus livré à son autorité, ce qu'il a fait, et ce qu'il est advenu de lui: le Rapport de Pilate à Tibère a la particularité de ne pas s'arrêter à la crucifixion mais de faire de Pilate un témoin direct de la résurrection du Christ. Ce texte est sans doute l'expression d'un membre d'une communauté qui considère Pilate d'un œil «non défavorable ${ }^{1} » ;$ mais du Rapport de Pilate à Tibère, on ignore l'auteur, la date et les circonstances d'écriture. C'est un texte agréable à lire, qui essaie de proposer le point de vue du plus célèbre des gouverneurs romains, et qui nous renseigne sur l'imaginaire apocalyptique d'une communauté chrétienne; c'est un récit plein d'humanité qui présente Jésus comme un admirable thaumaturge, et qui propose une description animée de phénomènes surnaturels; c'est enfin un écrit qui a été jugé digne d'être transmis de manuscrit en manuscrit au Moyen Âge, et dont j'espère présenter ici les différents témoins².

Les références associées aux deux principales recensions du Rapport de Pilate à Tibère dans la Bibliotheca Hagiographica Graeca ${ }^{3}$ et dans la Clavis

1. C'est la litote qu'emploie A. WALKER, «The Report of Pilate», dans Anti-Nicene Christian Library: Translations of the writings of the fathers down to A.D. 325, t. 16: Apocryphal Gospels, Acts, and Revelations, A. Roberts-J. Donaldson (dir.), Edinbourgh, 1870, 1951, p. 12: «It has been well remarked $(\ldots)$ that the early Church looked on Pilate with no unfavourable eye; that he is favourably shown in the catacombs; that the early Fathers interpreted him as a figure of the early Church, and held him to be guiltless of Christ's death; that the Creed does not condemn him, and the Coptic Church has even made him a saint. »

2. On peut lire une traduction en français du Rapport de Pilate d̀ Tibère (recension $\mathbf{B}$, d'après l'édition de J. C. ThILO, Codex Apocryphus Novi Testamenti, Leipzig, 1832, p. 803-813) de R. GOUNELle dans Écrits apocryphes chrétiens, t. 2, P. GEOLTRAIN-J.-D. KAESTLI (dir.), Paris, 2005, p. 311-317.

3. F. HALKIN, Bibliotheca Hagiographica Graeca, $3^{e}$ éd, Bruxelles, $1957\left[B H G^{3}\right]$; F. HALKIN, 
Apocryphorum Novi Testamenti ${ }^{4}$ sont BHG 779yI / CANT 66 pour la recension A, $B H G 779 x I / C A N T 65$ pour la $\mathbf{B}$. La recension $\mathbf{A}$ est généralement liée à la Comparution de Pilate, BHG 779yII / CANT 66, et la recension B à la Réponse de Tibère, BHG 779xII / CANT 65, mais ce n'est pas toujours le cas. En recherchant les manuscrits qui comportaient ces textes à l'occasion de mon travail de Master 2 , préparé à l'École Pratique des Hautes Études sous la direction du professeur JeanDaniel Dubois, j'ai mis au jour des témoins qui n'étaient pas recensés dans la $C A N T$, et j'ai pu aussi corriger quelques erreurs dans les références.

Pour présenter les manuscrits, je suis l'ordre de la CANT, et je présente d'abord la recension $\mathbf{B}$, à l'inverse donc de Tischendorf. Je reprends en revanche sa dénomination peu originale des recensions, $\mathbf{A}$ et $\mathbf{B}$. Dans son édition du Rapport de Pilate à Tibère publiée en $1853^{5}$, Tischendorf est le premier à attribuer une lettre aux quelques manuscrits qu'il a consultés. Il les nomme en suivant l'ordre alphabétique, et il semble pertinent de reprendre les lettres qu'il a données. Je donne la référence de chaque manuscrit en précisant entre crochets droits sa référence - fût-elle fautive - dans la CANT ${ }^{6}$. Je donne les numéros de folios correspondant au Rapport de Pilate à Tibère et non à l'ensemble composé du Rapport de Pilate à Tibère et de la Réponse de Tibère. En ce qui concerne les datations des manuscrits, j'apporte des informations plus récentes, issues des derniers catalogues, qui corrigent les renseignements de la $C A N T$ ou de la $B H G$ dont, quand cela est possible, je donne la référence, en précisant quelle édition, $B H G^{3}$ ou $B H G^{\text {na }}$, fait mention du manuscrit.

L'intérêt principal d'un travail sur les manuscrits est d'essayer de mettre en lumière les liens qui les unissent. Je n'entrerai pas ici dans le détail des relevés lexicaux qui permettent de corroborer telle ou telle hypothèse; je me permets toutefois de proposer des parentés entre certains manuscrits, au vu de l'environnement du Rapport de Pilate à Tibère tel que le décrivent les catalogues et en m'appuyant sur mes propres lectures des manuscrits?.

Novum Auctarium Bibliothecae Hagiographicae Graecae, Subsidia hagiographica 65, Bruxelles, $1984\left[B H G^{\text {na }}\right]$.

4. M. GeErard, Clavis Apocryphorum Novi Testamenti, Turnhout, 1992 [CANT].

5. K. TisCHENDORF, Evangelia Apocrypha, Leipzig, 1853, 21876, p. LXXVLI-LXXIX. J'ai consulté l'édition de 1876

6. Je choisis d'harmoniser la dénomination des manuscrits en donnant en français le nom de leur ville d'origine et en précisant aussi clairement que possible dans quelle bibliothèque ils se trouvent.

7. Pour plus de détails, je renvoie à mon mémoire de Master 2, «Édition et traduction d'un texte apocryphe grec: le Rapport de Pilate à Tibère», préparé à l'École Pratique des Hautes Études sous la direction du professeur Jean-Daniel Dubois, et disponible en version électronique à la bibliothèque de l'École Normale Supérieure, 45 rue d'Ulm, à Paris. 


\section{I. - TÉMOINS de LA RECENSION B DU RAPPORT DE PILATE Ȧ TIBERRE (CANT 65)}

\section{A. Les manuscrits édités par Tischendorf}

Tischendorf a consulté cinq manuscrits auxquels il a attribué une lettre en suivant l'ordre alphabétique. La $B H G$ renvoie aux éditions ${ }^{8}$; les indications de la CANT sont plus précises que celles de Tischendorf.

A Vienne, BN, theol. gr. 247, $2^{\text {e }}$ moitié du Xve s9., f. $148^{\mathrm{v}}-151$ (BHG $\left.779 \mathrm{xI}\right)$ [Vindobon. theol. gr. 246, XIV s., avec une erreur de numérotation de la CANT, 246 pour 247]

Ce manuscrit est daté dans le catalogue le plus récent de la deuxième moitié du XV s. Le Rapport de Pilate à Tibère y est précédé d'une «homélie sur la conception de la Mère de Dieu» de Jean d'Eubée et suivi d'une «homélie sur la dormition de Marie » de Jean de Thessalonique. Il est accompagné de la Réponse de Tibère, f. 151-152 $2^{v}$. Le texte de ce manuscrit a été reproduit par Birch ${ }^{10}$, et Thilo l'a utilisé par endroits, notamment pour compléter sa lecture du manuscrit de Paris $\mathrm{gr}$. $1019 \mathrm{~A}$. Tischendorf utilise aussi ce manuscrit, mais en se référant uniquement aux éditions de ses prédécesseurs, ce qui a entrainé la reproduction des quelques erreurs - d'importance mineure - commises par Birch.

B Turin, c. II. 5 (Pasini gr. 302), XII' s., f. $60^{\mathrm{v}}$ (BHG 3 779xI) [Taurin. gr. II, 5]

La référence de ce manuscrit est douteuse: dans l'inventaire de Pasini, en $1749^{11}$, le manuscrit porte bien le numéro 302, accompagné de la référence c. II. 5 ; mais dans l'inventaire des bibliothèques d'Italie, en $1922^{12}$, il est classé au

8. A. BIRCH, Auctarium Codicis Apocryphi Novi Testamenti Fabriciani, Copenhague, 1804, p. 160-171; J. C. ThlLo, Codex Apocryphus Novi Testamenti, Leipzig, 1832, p. 803-813; F. F. FLECK, Wissenschaftliche Reise durch das südliche Deutschland, Italien, Sicilien und Frankreich, t. 2, 3' partie, Leipzig, 1837, p. 143-145; K. TISCHENDORF, Evangelia Apocrypha, Leipzig, 1853, 21876, p. 435-449; J. B. AUfHAUSER, Antike Jesus-Zeugnisse, Kleine Texte für Vorlesungen und Übungen 126, Bonn, 1913, p. 34-36 dans BHG'; A. DE SANTOS OTERO, Los Evangelios Apócrifos, Madrid, 1956, p. 507-514 dans $B H G^{\text {na }}$. La première édition du Rapport de Pilate à Tibère est sans doute celle de J. A. FABRICIUS, Codex Apocryphum Novi Testamenti, t. 2, Hambourg, 1703, ${ }^{2} 1719$, p. 456-465.

9. H. HUNGER-W. LACKNER-C. HANNICK, Katalog der griechischen Handschriften der österreichischen Nationalbibliothek, 3/3: Codices theologici 201.337, Vienne, 1992.

10. A. BIRCH, Auctarium Codicis Apocryphi Novi Testamenti Fabriciani, p. 160: Pilati de Christo relatio ad Tiberium Caesarem ex Codice Caesareo Vindobonensi 246.

11. J. PASINI, Codices manuscripto bibliothecae regii taurinensis Athenaei, Turin, 1749, t. 1, p. $392, n^{\circ} 302$.

12. A. SORBELl, Inventari dei manoscritti delle Biblioteche d'Italia, t. 28; Torino, Florence, 1922, p. $24, \mathrm{n}^{\circ} 198$. 
numéro 198, accompagné de la référence B. V. 8 (et suivi de la mention «Pasini gr: $302 »)$. Il s'agit bien du même manuscrit, daté du XIIr" s. Le texte qui nous intéresse est précédé des quatre Évangiles et suivi d'un texte intitulé Fragmentum Chronicorum de genealogia Deiparae d'Hippolyte de Thèbes. Il est accompagné de la Réponse de Tibère, f. 61. Ce manuscrit a été édité en 1837 par F. F. Fleck ${ }^{13}$ qui le considérait comme une «nouvelle recension ${ }^{14}$ » du Rapport de Pilate à Tibère: de fait, le texte de ce manuscrit propose un ordre narratif différent des éditions précédentes, notamment de celle de Thilo à laquelle renvoie Fleck. Cet ordre, à ma connaissance, lui est propre. Il ressemble à celui du texte que l'on trouve dans ce que j'ai appelé le «deuxième groupe de la recension $\mathbf{B}$ » (voir plus bas) mais le vocabulaire et les tournures restent toujours plus proches du «premier groupe». Tischendorf a utilisé l'édition de Fleck.

C Venise, Bibl. marcienne, II, 42, XuI ${ }^{\mathrm{e}} \mathrm{XIV} \mathrm{V}^{\mathrm{e}}$ s., f. $130^{\mathrm{v}}-132\left(\mathrm{BHG}^{3} 779 \mathrm{xI}\right)$ [Venet. Marc. II, 42, XII ${ }^{\mathrm{e}}-\mathrm{XIII}{ }^{\mathrm{e}}$ s., f. $\left.130^{\mathrm{v}}-132\right]$

Dans ce manuscrit, le Rapport de Pilate à Tibère fait partie d'un ensemble d'écrits liés à la Semaine Sainte, essentiellement des homélies de Jean Chrysostome ${ }^{15}$. Il est immédiatement précédé d'une homélie sur la trahison de Judas, pour le Jeudi Saint, et suivi d'une homélie de l'évêque Eusèbe d'Émèse lue le Vendredi Saint. Notre texte, qui décrit les circonstances de la Crucifixion et de la Résurrection, $s$ 'intègre donc parfaitement à cet ensemble. Il est suivi sans solution de continuité de la Comparution de Pilate qui commence au f. 132 ( $B H G^{3} 779 y$ II). Le catalogue de Mingarelli date ce manuscrit des $\mathrm{XII}^{\mathrm{e}}-\mathrm{XIII}^{\mathrm{e}} \mathrm{s}$. (c'est l'indication que reprend la CANT) et celui de Mioni ${ }^{16}$ des XII" ${ }^{\mathrm{e}} \mathrm{XIV}^{\mathrm{e}} \mathrm{s}$. Tischendorf utilise ce manuscrit dans son intégralité ${ }^{17}$, mais en proposant parfois des leçons fautives.

13. F. F. FLECK, Wissenschaftliche Reise durch das südliche Deutschland, Italien, Sicilien und Frankreich, t. 2, 3 partie; le Rapport de Pilate à Tibère se trouve aux p. 143-145.

14. Ibid., p. XI.

15. G. L. Mingarelli, Graeci Codices manu scripto apud Nanios Patricios Venetos asservati, Bologne, 1784, p. $103: n^{\circ} 63$ (16) Jean Chrysostome, In ramos palmarum; (17) id., In parabolam de ficu arefacta (feria magna 2); (18) id., In decem virgines (feria magna 3); (19) Éphrem le Syrien, Sermo in mulierem peccatricem (feria magna 4); (20) Jean Chrysostome, De proditione Iudae sermo I (feria magna 5); (21) Anaphora et paradosis Pilati; (22) Eusèbe, In diabolum et orcum (parasceve magna); (23) Jean Chrysostome, In s. Parasceven et in passionem Domini (eadem die); (24) Épiphane de Chypre, In sabbato magno homilia 2 (sabbato magno); (25) Grégoire d'Antioche, In mulieres unguentiferas; (26) Grégoire de Nazianze, Homilia I, in sanctum Pascha.

16. E. MION!, Codices graeci manuscripti bibliothecae divi Marci Venetiarum, t. $1:$ Codices in classes a prima usque ad quintam inclusi, 1 tre partie : Classis I-Classis ll, Codd. 1-120, Rome, $1967,142(21)$.

17. K. TISCHENDORF, Evangelia Apocrypha, p. LXXXX, «C i.e. Venet. Marcian. class. II. cod. XLII, saeculi fere XII [...] Totum textum descripsimus. »

(1) Notition 1 1230.2

(c) mil

Pllatie a

consulte monusutit uet in a reprolit i: de contat Viente

1) Pontis, I Xives, 1

Comai de Pllate solarmm. a ete utili lul attrib lin linte de manusent

Le lex los mên deux ma justifier | recentom pom la d distingue ceux qui antour du dectils, a chante lat Cosmuni IIs a'cum avec lisp

18. 14 top partie

19.1 (')

20.4. tib: anctent 
D Paris, BNF, gr. 1019 A, XIV es., f. $236^{\vee}-237^{\mathrm{v}}($ BHG 3 779xI) [Paris. B.N. gr. 1019, f. $236^{\left.\mathrm{v}-237^{\mathrm{v}}\right]}$

Ce manuscrit du XIV ${ }^{e}$ s. contient 237 folios et se termine par le Rapport de Pilate à Tibère qui clôt une série de dix lettres de Jean Chrysostome ${ }^{18}$. Il a été consulté par Thilo ${ }^{19}$ qui note ses points communs avec le manuscrit $\mathrm{A}$ et avec le manuscrit E. Malheureusement, le manuscrit est incomplet; le texte s'arrête avec

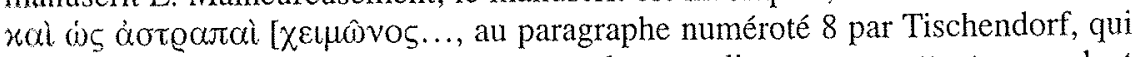
reprend ici le travail de Thilo. Les pages dont on dispose permettent cependant de constater la forte proximité de ce texte avec celui proposé par le manuscrit de Vienne, theol. gr. 247 (A).

E Paris, BNF, gr. 1331 , XIV ${ }^{e}$ s., f. $467-468^{\vee}$ (BHG $\left.779 x \mathrm{x}\right)$ [Paris. B.N. gr. 1331, XIV's., f. 467-468]

Ce manuscrit du XIV e s. portait autrefois la cote Mazarin.-Reg. 2504. Le Rapport de Pilate à Tibère s'y trouve entre un traité intitulé Methodius inveniendi cyclum solarem et lunarem et un traité de saint Maxime, De statu ecclesiae catholicae ${ }^{20}$. Il a été utilisé par Thilo, qui lui attribue la lettre C, et via Thilo par Tischendorf, qui lui attribue la lettre $\mathrm{E}$. Ce texte est mentionné par la CANT au paragraphe 65 , dans la liste des cinq manuscrits de Tischendorf, mais aussi au paragraphe 66, parmi les manuscrits non édités. Sans doute est-ce par erreur.

Le texte du Rapport de Pilate à Tibère proposé par ce manuscrit présente les mêmes particularités de récit que celui du manuscrit de Venise, $I I, 42$. Ces deux manuscrits, ainsi que d'autres que je mentionne plus bas, me paraissent justifier la nécessité de créer, à l'intérieur de la famille de manuscrits dits «de la recension $B$ », un groupe caractérisé par un ordre narratif différent, notamment pour la deuxième partie du texte: alors que certains manuscrits de la recension $\mathbf{B}$ distinguent clairement les phénomènes accompagnant la crucifixion de Jésus de ceux qui accompagnent sa résurrection, d'autres organisent cette partie du récit autour du lien entre la crucifixion et les phénomènes sismiques et météorologiques décrits, avant de mentionner l'apparition dans le ciel lumineux de la foule qui chante la gloire de Dieu et les secousses qui précèdent le cortège des patriarches. Ces manuscrits font disparaître toute mention explicite de la résurrection du Christ. Ils n'évoquent pas non plus les juifs qui périrent cette nuit-là. La narration reprend avec l'épisode de l'engloutissement des synagogues.

is Venetos asserum; (17) id., In magna 3); (19) Thrysostome, $D e$ (22) Eusèbe, In let in passionem abbato magno); ze, Homilia 1 , in

, t. 1 : Codices in d. 1-120, Rome, Ircian. class. II.
18. H. OMONT, Inventaire sommaire des manuscrits grecs de la Bibliothèque nationale, fere partie: ancien fonds grec, théologie, Paris, 1886, p. 204, $\mathrm{n}^{\circ} 1019 \mathrm{~A}$.

19. J. C. THLO, Codex Apocryphus Novi Testamenti, p. 103.

20. 1. OMONT, Inventaire sommaire des manuscrits grecs de la Bibliothèque nationale, $2^{\circledR}$ parlie: ancien fonds grec, droit, histoire, science, Paris, 1888, p. 12 13. 
Outre leurs nombreux points communs lexicaux, les manuscrits de ce groupe - que je désigne comme «deuxième groupe», car il me semble minoritaire partagent l'utilisation de la deuxième personne du pluriel pour s'adresser à l'empereur. Ils proposent un texte plus court que ceux du premier groupe. À cela s'ajoute que quatre manuscrits, sur les cinq que j'ai identifiés comme appartenant à ce groupe, sont suivis de la Comparution de Pilate, et non de la Réponse de Tibère $^{21}$. Je préciserai ces éléments au cours de la description des manuscrits.

\section{B. Manuscrits identifiés mais non édités}

Parmi les manuscrits cités sous la référence CANT 65, les manuscrits suivants n'ont pas encore été édités:

Athos, Grande Laure, K. 64, 1634, f. 91-95 [Athon. Laurae 64, XV s., f. 91-99, Réponse de Tibère comprise]

Le catalogue des manuscrits de la Grande Laure du Mont Athos, qui précise la date de ce manuscrit, signale que le Rapport de Pilate à Tibère y est suivi de la Réponse de Tibère ${ }^{22}$. L'ensemble est encadré de deux lettres, l'une de Grégoire de Rome, et l'autre de Basile de Césarée. Il n'y a pas d'autre «lettre» dans ce manuscrit. L'Institut de Recherche et d'Histoire des Textes, section grecque, possède la copie de la première page du texte, qui se révèle à la lecture assez proche des manuscrits de Turin c. II, 5 (302), de Londres, Harley 5556 et Burney 342, et de Paris suppl. gr. 78; il appartiendrait plutôt au premier groupe.

Vatican, Bibl. vaticane, gr. 417, XII s., f. $365^{\mathrm{v}}-366$ [Vat. gr. 417 , XIr s., f. $365^{\mathrm{v}}$ $-366]$

Dans le manuscrit du Vatican $g r .417$ (anciennement 286), la recension B du Rapport de Pilate à Tibere ${ }^{23}$ est suivie sans solution de continuité par la Comparution de Pilate, dans les folios 366-367. Le texte est précédé d'homélies de saint Basile et suivi du récit de la vie et des miracles de saint Basile. On ne s'explique pas cette intrusion, dans un ensemble de textes relatifs à un même saint, du Rapport de Pilate à Tibère. Ce manuscrit, que je n'ai pu consulter, semble être le plus ancien témoin du Rapport de Pilate à Tibère.

21. Les quatre manuscrits sont ceux de Venise, Bibl. marcienne, $I l, 42$, de Madrid, Bibl. de l'Escurial, $\omega I V, 18(570)$, d'Oxford, Bibl. bodléienne, Lincoln 1, et de Patmos, Monastère de Saint Jean le Théologien, 448. Le cinquième, le manuscrit de Paris, BNF, gr. 1331, n'est lié à aucun texte relatif à Pilate.

22. S. EUSTRATIADES-SPYRIDON LAURIOTES, Catalogue of the Greek Manuscripts in the Library of the Laura on Mount Athos, Cambridge, Mass., 1925, p. 227, $\mathrm{n}^{\circ} 1351$ (7).

23. R. Devreesse, Codices Vaticani Graeci, t. 2 : codices 330-603, Vatican, 1937, p. 128 (2).

fondres. 111

(1.29) 21

1.c catcilou 1711 Notre procede d'un Christas est Aethighatal: mantuscrit sui

Puris, BNI, I. 200.201

Dins co m de lesu Chrt Il ost suivi d to monuscrit d'Omont, me munuscrits de Londres Birt

Athenes, BN Alhen. BN

Leti matulu: Sépulcre) d" d'Athenes, 5 du culalog Merohtion al Reponse de penter qu'il: Vienne $(A)$.

24. A Catal p. $276,0^{10} 35$

35. Poutedre de la souda (e) $(\mathrm{tr}), \mathrm{t}, 2.0 .75$

20. $1 \mathrm{dmm}$.

$29.110 \mathrm{MO}$ liat: anclen font p. $214.11^{8} 72$

28. 1611p://min

20, I M. () Marref Richar 


\section{'ERINE BAUDOIN}

scrits de ce groupe nble minoritaire pour s'adresser à lier groupe. À cela omme appartenant de la Réponse de les manuscrits.

lanuscrits suivants

4, $X V^{e}$ s., f. 91-99,

hos, qui précise la $e$ y est suivi de la l'une de Grégoire ; «lettre» dans ce tion grecque, posture assez proche et Burney 342, et ..

$17, \mathrm{XII}^{\mathrm{c}}$ s., f. $365^{\mathrm{v}}$

, la recension $\mathbf{B}$ continuité par la ścédé d'homélies int Basile. On ne à un miême saint, ulter, semble être

de Madrid, Bibl. de , Monastère de Saint 'I, n'est lié à aucun

Manuscripts in the $51(7)$.

1, 1937, p. $128(2)$
TEMOINS MANUSCRITS DU RAPPORT DE PILATE À TIBÈRE

Londres, BL, Harley 5556, 1714, f. 29-30 [Londin. Brit. Libr. Harl. 5556, 1714 , f. 29.32]

Le catalogue de la collection Harley indique que ce manuscrit a été copié en 1714. Notre texte y est accompagné de la Réponse de Tibère, f. 31-3224. Il est précédé d'un texte dont le titre en latin est Historia quam habent Judaei, quod Christus est filius Dei: cujus et meminit Suidas ${ }^{25}$, et suivi d'un texte intitulé Aenigmata Patriarchae Alexandriae. Il est très proche du texte proposé par le manuscrit suivant.

Paris, BNF, suppl. gr. 78, XVIII ${ }^{\mathrm{e}}$ s., f. 200-202 [Paris. B.N. suppl. gr. 78, XVII ${ }^{\mathrm{e}}$ s., 1. 200-203]

Dans ce manuscrit, le Rapport de Pilate à Tibère est précédé d'un texte intitulé de Jesu Christo, ex Suida ${ }^{26}$, accompagné de la Réponse de Tibère, f. 202-203, et il est suivi des Gerasimi Patriarchae aenigmata ${ }^{27}$, les mêmes textes que dans le manuscrit Harley 5556. Le manuscrit est daté du XVII ${ }^{\mathrm{e}} \mathrm{s}$. dans le catalogue d'Omont, mais du XVIII $\mathrm{s}$. sur la base iconographique en ligne du département des manuscrits de la $B N F^{28}$. Il est probable que ce manuscrit ait copié le manuscrit de Londres Burney 342.

Athènes, BN, autrefois Métochion du Saint-Sépulcre 303, XIV $-\mathrm{XV}^{\mathrm{e}}, \mathrm{f} .158-159^{\mathrm{v}}$ [Athen. B.N. olim Methochii 303, $\mathrm{XIV}^{\mathrm{e}}-\mathrm{XV}^{\mathrm{e}}$, f. 158-159v]

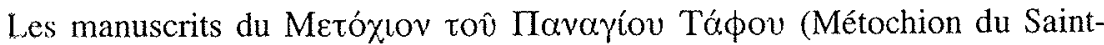
Sépulcre) d'Istanbul sont aujourd'hui conservés à la bibliothèque nationale d'Athènes, comme l'indique J.-M. Olivier ${ }^{29}$. Ils sont décrits dans les tomes 4 et 5 du catalogue des manuscrits de Jérusalem de Papadopoulos. Dans le manuscrit Métochion du Saint-Sépulcre 303, le Rapport de Pilate à Tibère est suivi de la Réponse de Tibère; la recension n'est pas indiquée, mais le titre donné laisse penser qu'il s'agit bien de la recension $\mathbf{B}$ : il est semblable à celui du manuscrit de Vienne (A). Le Rapport de Pilate à Tibère et la Réponse de Tibère sont encadrés

24. A Catalogue of the Harleian Manuscripts in the British Museum, Londres, 1808-1812, t. 3, p. $276, n^{\circ} 5556(3)$.

25. Peut-être la version brève du texte intitulé Sur le sacerdoce du Christ, inséré dans le lexique de la Souda (cf. Sur le sacerdoce du Christ, dans Écrits apocryphes chrétiens, F. G. NuvolonE (tr.), t. 2, p. 75-99).

26. Idem.

27. H. OMONT, Inventaire sommaire des manuscrits grecs de la Bibliothèque nationale, $3^{\mathrm{e}}$ parlic: ancien fonds grec, belles-lettres, Coislin, suppléments, Paris et départements, Paris, 1888 , p. $214, \mathrm{n}^{\circ} 78$.

28. http://mandragore.bnf.fr:

29. J.-M. OLIVIER, Répertoire des bibliothèques et des catalogues de manuscrits grecs de Marcel Richard, Turnhout, ${ }^{3} 1995$, p. 109. 
par deux textes qui ne sont apparemment liés ni à la Passion ni à Pilate, une ode de Léon le Sage et un discours de Basile de Césarée.

Ce manuscrit semble être répertorié deux fois dans la $C A N T$, dans le groupe des manuscrits contenant la recension $B$, la première fois sous la référence Athen. B.N. olim Metochiii 303, XIV $-\mathrm{XV}^{\mathrm{e}}$ S., f. 158-159 et la seconde fois comme Hierosol. Crucis $303, \mathrm{XIV}^{\mathrm{e}}-\mathrm{XV}$ s., f. $153-160^{\mathrm{v}}$. Or aucun manuscrit du fonds $\tau$ ố Tutiov

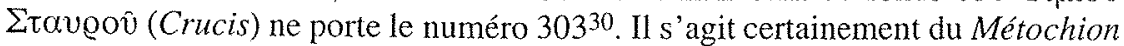
du Saint-Sépulcre 303. En outre, les références des folios sont proches et la CANT date les deux manuscrits de la même époque.

Milan, Bibl. ambrosienne, $H 22$ sup. (426), XV s., f. 7-7v [Mediol. Ambr: H 22 sup. (426), XV s., f. 132-133]

La recension $\mathrm{B}$ du Rapport de Pilate à Tibère se trouve dans ce manuscrit au folio 7 , et non $\mathrm{f} .132-133^{\circ}$ comme l'indique la CANT ${ }^{31}$. Elle est suivie de la Réponse de Tibère (f. 8), et l'ensemble est précédé d'un texte intitulé $\theta \varepsilon o \tau o x i ́ a$, $<$ Manuelis $>$ Philae Carmina et suivi d'une réflexion de fructu quem lectio librorum sanctorum affert. Le copiste semble avoir été quelque peu distrait dans son travail, et le texte qu'il propose comporte de nombreuses omissions et lectures propres qui laissent penser que cette version du Rapport de Pilate à Tibère n'a pas servi de modèles à d'autres copies.

M. Geerard cite aussi le manuscrit Paris. B.N. suppl. gr. 1169,1685, f. $43^{\mathrm{v}}-46^{\mathrm{v}}$, or le texte désigné par cette référence est uniquement la Réponse de Tibère. Le Rapport de Pilate à Tibère le précède bien dans ce manuscrit, mais il s'agit du texte de la recension dite A par Tischendorf, CANT 66, aux folios 39-43. Quant au manuscrit Paris. B.N. gr. $1771, \mathrm{XV}^{\mathrm{e}}$ s., f. $282^{\mathrm{Y}}-283$, cité aussi par M. Geerard, il ne contient que la Réponse de Tibère, et non le Rapport de Pilate à Tibère.

\section{Manuscrits mal identifiés}

Certains manuscrits ont en revanche été classés en CANT 66 alors qu'ils contiennent un texte proche de celui des manuscrits de CANT 65.

Patmos, Monastère de saint Jean le Théologien, 448, XV s. , f. 61-63 [Patm. 448, $\mathrm{XV}^{\mathrm{e}}$ s., f. 61-65v]

Ce manuscrit est sans doute mentionné par M. Geerard parmi les manuscrits de CANT 65, appartenant à la recension $\mathbf{A}$, parce qu'il est suivi, sans solution de continuité, de la Comparution de Pilate, mais le texte du Rapport de Pilate

30. Ibid., p. 395.

31. E. MARTINI-D. BASSI, Catalogus codicum graecorum Bibliothecae Ambrosianae, t. 1 , Milan, 1906, p. 505, H 22 Sup (426). 


\section{'HERINE BAUDOIN}

I ni à Pilate, une ode

$\Gamma$, dans le groupe des śférence Athen. B.N. is comme Hierosol. i fonds tov Tuúov sment du Métochion proches et la $C A N T$

Mediol. Ambr. H 22

dans ce manuscrit Ille est suivie de la intitulé $\theta \varepsilon 0 \tau o x i ́ \alpha$, a quem lectio librosu distrait dans son aissions et lectures ate à Tibère n'a pas

9,1685, f. $43^{\mathrm{v}}-46^{\mathrm{v}}$, onse de Tibère. Le t, mais il s'agit du lios 39-43․ Quant par M. Geerard, il tte à Tibère.

$T 66$ alors qu'ils $i 5$.

51-63 [Patm. 448, mi les manuscrits ivi, sans solution Rapport de Pilate

: Ambrosianae, t. 1, a Tibère qu'il contient est plus proche du «deuxième groupe» de la recension $\mathbf{B}$. Dans le catalogue des manuscrits du monastère de Patmos ${ }^{32}$, une note indique l'incapacité de l'auteur du catalogue à dire à quelle édition existante le texte est semblable; il en cite donc l'incipit ${ }^{33}$. La consultation de la copie de ce manuscrit à I'IRHT permet de constater que le texte appartient à la recension $\mathrm{B}$. Le Rapport de pilate à Tibère est précédé de la Vie de Grégoire d'Assos et suivi d'un traité ano-

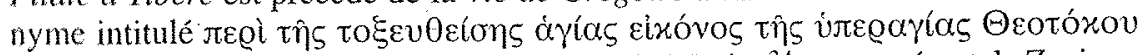

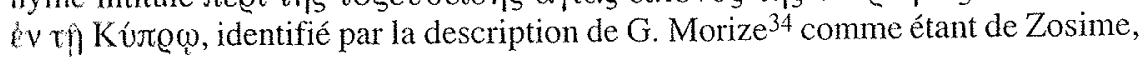
BHG 1890d.

Londres, BL, Add. 25881, XVI ${ }^{\mathrm{e}}$ s., f. $255-257^{\vee}$ (BHG 7797b) [Londin. Brit. Libr. Add. 25881, $\mathrm{XVI}^{\mathrm{e}}$ S., f. 255-260v]

Dans ce manuscrit, le Rapport de Pilate à Tibère est suivi de la Réponse de Tibere; on ne voit donc pas ce qui motive son classement par M. Geerard parmi les manuscrits de CANT 66. Ce manuscrit contient des écrits de natures diverses, hagiographies, canons, homélies, hymnes et lettres, écrits sur une seule colonne. II est téférencé comme $B H G 779 \mathrm{zb}$, Anaphorae et Paradosis Pilati alia recensio; de fait, le texte est caractérisé par de nombreuses formules qui lui sont propres, des ajouts et des reformulations. Le Rapport de Pilate à Tibère est précédé des Oracles de Léon le Sage, et est suivi de la Réponse de Tibère ${ }^{35}$, qui est le dernier texte du manuscrit.

La lecture de ce manuscrit met en lumière la grande proximité de son texte ivec celui qui est proposé par le manuscrit de Milan $H 22$ sup. (426), bien que le copiste semble avoir largement remanié le texte. Je fais l'hypothèse d'une source commune aux manuscrits de Londres Add. 25881, et de Milan H 22 sup. (426).

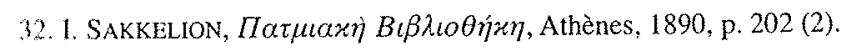

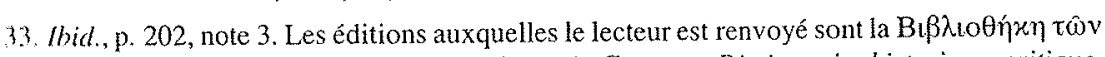
IIoreg(t)v, t. 7, à laquelle je n'ai pu avoir accès, et A. CALMET, Dictionnaire historique, critique, 'hronologique, géographique et littéral de la Bible, t. 3: N-T, Paris, 1730, s. v, «Pilate», p. 230 : Calmet cite l'intégralité de la Lettre de Pilate à Claude, en traduction, et évoque «une autre lettre prétendue de Pilate à Tibère» dont il précise que «M. Fabricius en a donné une en grec, qui est lirée d'un manuscrit de $\mathrm{M}$. de Colbert, coté 2493 [manuscrit A de la recension A], et M. Cotelier en cite une autre, qui est aussi en Grec dans un manuscrit de la bibliothèque du Roi, $n^{\circ} 2431$ \%. Ce deuxième manuscrit correspond au manuscrit de Paris, BNF, gr. 854, selon H. OMONT, Inventaire wnmaire des manuscrits grecs de la Bibliothèque nationale et des autres bibliothèques de Paris 'f de's départements, Paris, 1898 , p. LXIX ; mais je n'ai pas pu y retrouver le Rapport de Pilate à Fiber re qui, dans le catalogue, n'est pas mentionné dans la description (H. OMONT, Inventaire som. marè des manuscrits grecs de la Bibliothèque nationale, Itre partie: ancien fonds grec, théologie, p. 159.160 ), et que je n'ai pas vu en feuilletant le manuscrit.

34. Feuillet dactylographié consulté à l'IRHT.

35. (atalogue of the Additions to the Maruscripts in the British Museum in the years 1854 $1,4 \%$. 1. 2. p. 229, Add. 25881 (7). 
L'Escurial, Bibl. royale, $\omega I V .18(570)$, XV s. s. f. $103^{\vee}-105$ [Scorial. $\omega$ IV.18 (570), $x v^{e}$ s., f. 103-105]

À propos de notre texte, le dernier du manuscrit, le catalogue de la bibliothèque de l'Escurial donne une référence confuse, précisant qu'il s'agit de la recension $\mathbf{B}$ tout en donnant les références des pages de la recension $\mathbf{A}$ chez Tischendorf ${ }^{36}$. Il s'agit bien de la recension $\mathbf{B}$. Le texte est suivi sans solution de continuité par la Comparution de Pilate qui clôt le manuscrit. Sa très grande proximité tout au long du texte avec le manuscrit de Venise $I I, 42$, laisse penser qu'il en est la copie. Il appartient donc au deuxième groupe de la recension $\mathbf{B}$.

\section{Nouveaux témoins de la recension $B$ (CANT 65)}

À ces témoins recensés par $\mathrm{M}$. Geerard, j'ajoute les sept manuscrits que mes recherches dans différents catalogues et index m'ont permis de mettre au jour ${ }^{37}$. Certains sont assez tardifs, mais ne sont pas sans importance dans l'histoire de la transmission du texte.

\section{Londres, BL, Burney 342, 1709, f. 91-94 (anc. 177-183)}

Le codex qui contient une version du Rapport de Pilate à Tibère est composé de deux manuscrits, le premier du XV $\mathrm{XV}^{\mathrm{e}}$ s. et le second daté de 1709 et copié par un diacre du nom de Nicodème. Le premier texte du second manuscrit est une homélie du patriarche Gerasime d'Alexandrie intitulé Sermo contra Romanos de Eucharistiae elementis; il est suivi du Rapport de Pilate à Tibère, sous le titre Pontii Pilati Epistola ad Tiberium Caesarem Imperatorem de Salvatore nostro Jesu Christo, aux f. 91-9438 (anciennement 177-183), accompagné de la mention barbaro-graece, comme les autres textes de ce manuscrit. Notre texte est accompagné de la Réponse de Tibère, f. $94^{\vee}$ à $96^{\vee}$, elle-même suivie d'une lettre du copiste, Nicodemi Diaconi (...) epistola brevis ad Arsanium Metropolitam Thebaidos.

Le texte du manuscrit est extrêmement proche de celui de Paris Suppl. gr. 78. En outre, le Rapport de Pilate à Tibère est, dans ce manuscrit de Paris, suivi d'un texte intitulé Gerasimi Patriarchae aenigmata, et dans le Inanuscrit de Londres Harley 5556, des Aenigmata Patriarchae Alexandriae, sans doute le même texte que celui qui précède le Rapport de Pilate à Tibère dans le manuscrit de Londres Burney 342. Je fais l'hypothèse d'une source commune aux manuscrits de Paris Suppl. gr. 78, et de Londres, Burney 342 et Harley 5556; cette hypothèse se

36. G. DE ANDRÉs, Catálogo de los Códices Griegos de la real Biblioteca de el Escorial, t. 3 : códices 421-570, Madrid, 1967, p. 225, n $570(6)$.

37. L'un d'eux, le manuscrit d'Oxford, Bibl, bodléienne, Lincoln $l$, est mentionné dans la $B H G^{\text {na }}$.

38. A Catalogue of Manuscripts in the British Museum, t. 1, $2^{\mathrm{e}}$ partie, the Burney Mantuscripts, p. 92 , Burn. 342 (4). 
l. $\omega I V .18(570)$

la bibliothèque de la recension $\because$ Tischendorf ${ }^{36}$. e continuité par oximité tout au en est la copie.

\section{)}

uscrits que mes lettre au jour ${ }^{37}$. l'histoire de la

re est composé 39 et copié par nuscrit est une a. Romanos de $e$, sous le titre !vatore nostro né de la menVotre texte est ie d'une lettre Metropolitam

Suppl. gr. 78. inis, suivi d'un at de Londres le même texte rit de Londres scrits de Paris hypothèse se

el Escorial t. 3 : intionné dans la ey Manuscripts, trouve confirmée par la lecture des manuscrits. Il est possible que la source de ces manuscrits soit à chercher dans le manuscrit de l'Athos K. 64 .

\section{Jutuntem, Patriarcat, Saint-Sépulcre 238, XVIII s., f. $62^{\mathrm{v}}-64^{\mathrm{v}}$}

Duns le catalogue des manuscrits de Jérusalem, il est fait mention d'un texte qui pourrait bien être le Rapport de Pilate à Tibère. La description est la sui-

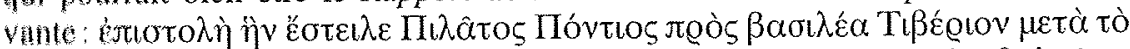

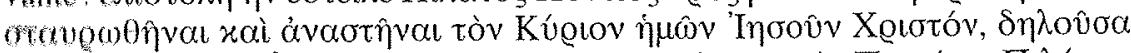

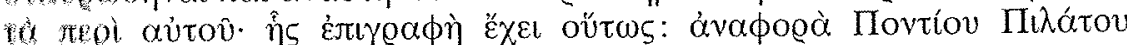
MYúvos 'lovdalas ${ }^{39}$, ce qui correspond au début de notre texte dans la recenNon $\mathrm{B}$, et particulièrement, parmi les manuscrits que j'ai consultés, au manuscrit

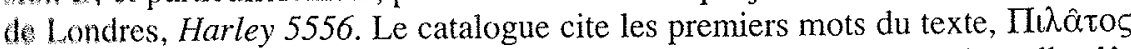

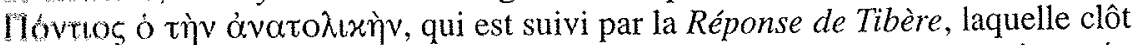
10 manuscrit. Le Rapport de Pilate à Tibère est précédé d'un récit intitulé lotogía.

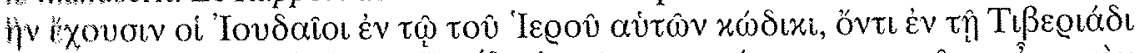

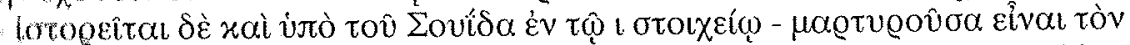

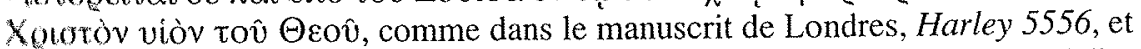
duns le manuscrit de Paris, Suppl. gr. 78. Ce manuscrit est donc certainement à lier מux deux manuscrits de Londres, Harley 5556 et Burney 342, et au manuscrit de Puris Suppl.gr. 78.

Altos. Grande Laure, M. 68, 1772, f. 208-209v

Le catalogue de la Grande Laure de l'Athos fait mention de deux manuscrits contenant le Rapport de Pilate à Tibère. Le manuscrit M. 68 de la Grande Laure

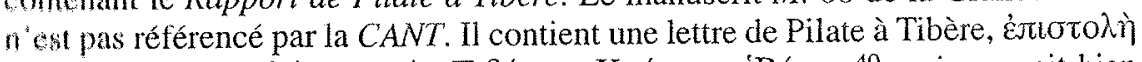

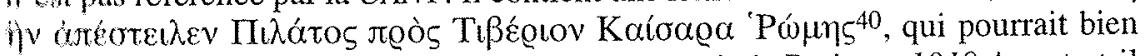
Gre le Rapport de Pilate à Tibère: ainsi le manuscrit de Paris gr. $1019 \mathrm{~A}$ porte-t-il lui aussi un titre dans lequel le terme áva crit, notre texte est précédé de vers de Gennade et suivi d'un texte intitulé Jóv

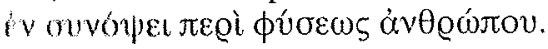

Alhenes, BN, 432 [130], XVI ${ }^{\mathrm{e}}$ s.

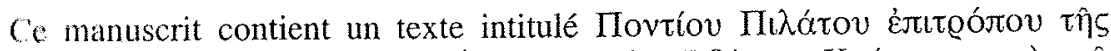

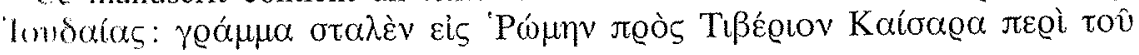

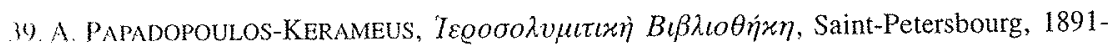
11) 15,1 . 1, p. $313, n^{\circ} 238(6)$.

10. S. EUSTratiades-SPYRIDON LAURIOTES, Catalogue of the Greek Manuscripts in the I ihrary of the Laura on Mount Athos, p. 315, $\mathrm{n}^{\circ} 1759$ (20).

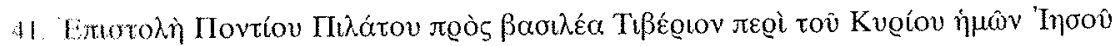
Sourrot 
Xouøtô̂ ${ }^{42}$. C'est exactement le même titre que celui que donne le manuscrit

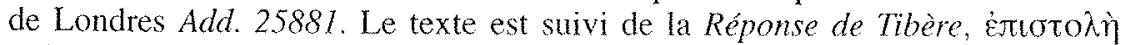

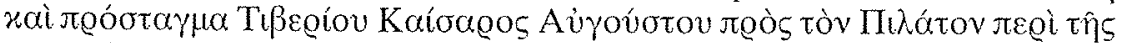

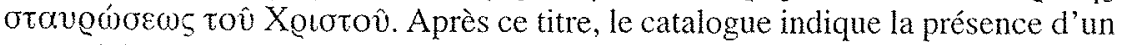

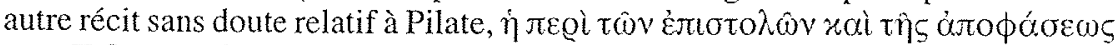

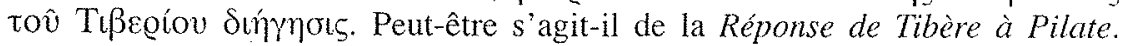
L'ensemble est précédé d'un texte d'Athanase d'Alexandrie, Joò $\varsigma$ 'Avtíoxov

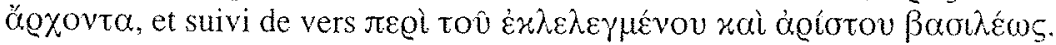

\section{Athènes, BN, 2069, XVIIr-XIX' s., f. 96-104 (BHG 779xI)}

Ce manuscrit est accompagné dans le catalogue de Politis de la référence $B H G$ 779xI-II. Il s'agirait donc de la recension $\mathrm{B}$ du Rapport de Pilate à Tibère suivie de la Réponse de Tibère, comme le laisse supposer le titre donné par le cata-

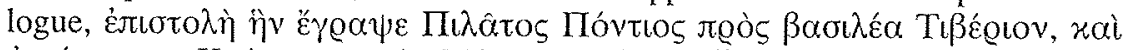

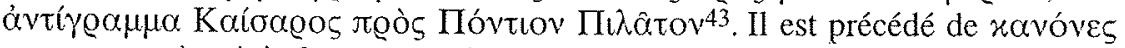

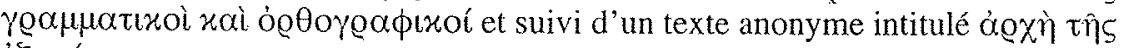
$\dot{\varepsilon} \xi \eta \gamma \eta \dot{\emptyset} \sigma \omega \varsigma$.

\section{Oxford, Bibl. bodléienne, Lincoln 1, $2^{\mathrm{e}}$ quart du XVe s., f. $4^{\mathrm{v}}-5^{\mathrm{v}}(B H G 779 \mathrm{z}$ ?)}

Ce manuscrit est considéré comme un epitome et classé $B H G 779 \mathrm{z}$ dans $B H G^{\text {na }}$, avec la mention des. mutil., alors que dans $B H G^{3}$, la cote $B H G 779 \mathrm{z}$ décrivait la version brève du Rapport de Pilate à Tibère éditée par G. F. Abbott. Ce rapprochement est inapproprié: le texte du manuscrit d'Oxford n'est pas un résumé, mais un témoin du «deuxième groupe » des manuscrits présentant la recension $\mathbf{B}$. En outre, il est complet. Il est regrettable que le catalogue édité récemment par le. Corpus der byzantinischen Miniaturenhandschriften indique qu'il s'agisse uniquement de la Comparution de Pilate ${ }^{44}$; un catalogue plus ancien ${ }^{45}$ cite quelques mots qui appartiennent à la fin du Rapport de Pilate à Tibère et qui sont les mêmes que dans le manuscrit de Venise $I I, 42$. Le Rapport de Pilate à Tibère est précédé de divers fragments ${ }^{46}$ et suivi d'homélies pour l'année liturgique, notam-

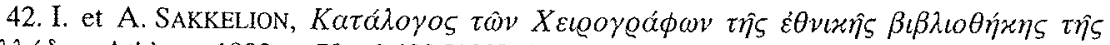

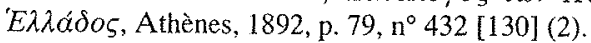

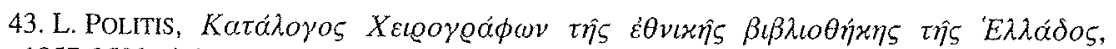
do. 1857-2500, Athènes, 1991, p. 108, n² 2069 (3).

44. I. HuTtER, Corpus der byzantinischen Miniaturenhandschriften, t. 5, $1^{\text {ire }}$ partie: Oxford College Libraries, Stuttgart, 1997, p. 63, $\mathrm{n}^{\circ}$ 26, Lincoln College gr. 1 (D.1) (1): «Paradosis Pilati, Exzerpt, des. mut. ».

45. H. O. CoXE, Catalogus codicum manuscriptorum qui in collegiis aulisque Oxoniensibus hodie adservantur, $1^{\text {ese }}$ partie : Catalogus codicum manuscriptorum collegii Lincolniensis, Oxford,

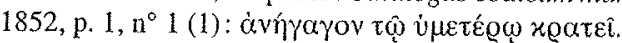

46. I. HUTTER, Corpus der byzantinischen Miniaturenhandschriften, t. $5,1^{\text {ère }}$ partie, p. 63 ,

mis:m fruth |s

$1: 10,10$

listosenthen

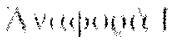

Mildang Ima lit latil yule to follos all peull lire rener revory

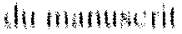

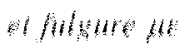

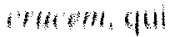

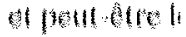
का In⿴囗十)

Mulan, $B(b)$ (111017901

I.tomingen ab mutusent fisyotit don logene indigu Acmothous 0.41 111

\section{Temon}

Sous rette reontand $A$ dre poufquo Cotle reches

A Path BN 1. 2) 294

C'manum! Guth attofol

$11^{28} 26(1): 41$

1. . |

a) $1 / \% h .1 !$

at $c$ bat? f. 411 
le donne le manuscrit

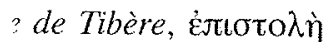

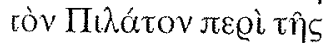
dique la présence d'un

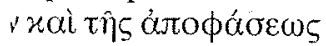
se de Tibère à Pilate.

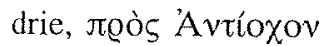
lotov $\beta \alpha \sigma \lambda \lambda \varepsilon ́ \omega \zeta$.

s de la référence $B H G$ Pilate à Tibère suivie re donné par le cata-

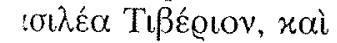
précédé de жavóves

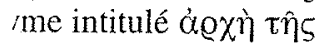

\section{$1-5^{\mathrm{v}}(B H G 779 z ?)$}

$4 G 779 z$ dans $B H G^{\mathrm{na}}$, :HG 779z décrivait la F. Abbott. Ce rappron'est pas un résumé, antant la recension $\mathbf{B}$. lité récemment par le te qu'il s'agisse unincien $^{45}$ cite quelques ibère et qui sont les te Pilate à Tibère est ée liturgique, notam-

$v \varkappa \hat{\eta} \varsigma \beta \iota \beta \lambda\llcorner o \theta \dot{\eta} \approx \eta \varsigma \tau \hat{\eta} \varsigma$

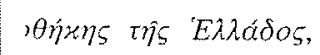
1. 5, 1 ${ }^{\text {irc }}$ partie: Oxford 1) (1): «Paradosis Pilati,

: aulisque Oxoniensibus ii Lincolniensis, Oxford,

t. 5, 1're partie, p. 63, munt pour le Carême et le temps pascal, comme dans le manuscrit de Venise ${ }^{47}$. Le toxte est suivi par la Comparution de Pilate mais la distinction entre les deux

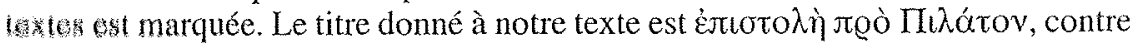

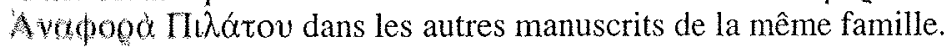

Ga description dans la $B H G$, qui pourrait être remise en cause, a pu être troublée pul la fat que le texte, écrit sur deux colonnes, est barré d'une grande croix en $x$, sur 16. follo $55^{v}$ et sur la partie du folio $4^{v}$ qui contient le Rapport de Pilate à Tibère: on peut lire en haut du folio 5 la note $\psi \varepsilon v \delta \circ \zeta \lambda_{0<\gamma \circ \zeta>\beta \lambda \alpha \sigma \phi \eta \mu \rho \zeta} \varepsilon \gamma \varrho \alpha \phi \eta$.

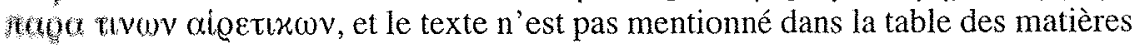
du mumuscrit : in hoc libro continentur 1. Andrea cyjusdam et Epiphanis de fulmine

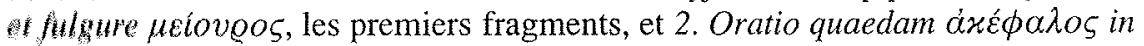
bucem, qui suit notre texte. Après le folio $5^{\mathrm{v}}$, c'est une main différente qui écrit, ot peut «tre les cinq premiers folios sont-ils d'un cahier différent. Enfin, le texte de of manuscrit partage des leçons propres aux manuscrits de Venise et de Madrid.

Milan, Bibl. ambrosienne, A 79 sup. (T 223, T 219, T 224), 1517, ff. $1-2^{v}$ $(10116779 \times 1)$

Le eatalogue des manuscrits hagiographiques de Milan signale la présence dans ce manuscrit du Rapport de Pilate à Tibère avec la référence $B H G 779 \times \mathrm{x}^{48}$. Il " viginat donc de la recension $B$ de notre texte, dont il manquerait la fin : le cata-

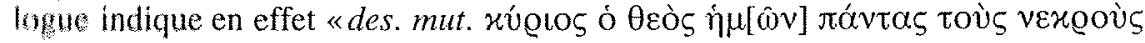

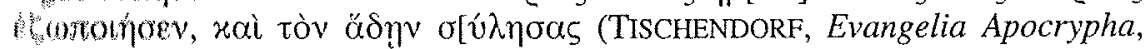
1. $(48,1,11) »$. Notre texte est suivi du menaeum novembris.

\section{ThMOINS DE LA RECENSION A dU RAPPORT DE PILATE À TIBËRE (CANT 66)}

$50 u s$ cette référence se trouve le texte que Tischendorf considère comme la feunsion A du Rapport de Pilate à Tibère, sans qu'il paraisse possible de comprendre pourquoi la $B H G$ puis la CANT n'ont pas suivi le même ordre de présentation. Culte recension du texte est souvent suivie de la Comparution de Pilate.

\section{A. Les manuscrits édités par Tischendorf}

A Puris, BNF, gr. 770, 1315, f. 25-27 (BHG 779yI) [Paris. B.N. gr. 770, 1315, 1. $25.29 \mathrm{~V}$

C t manuscrit comporte 268 folios. Il a été copié en 1315 par le prêtre Georges, et cuit autrefois référencé sous le nom Colbert 2493. Il est décrit de façon exhaustive

11 4 (1): Erbauliche Schriften, Fragment: [...] Vita Andreae stulti, [...] De fulmine, Exzerpte,

1 | lahua historia, Exzerpte : Daniel, Habakuk, Tobias [...]».

4) Ibid., p. 64, n $26(2)$.

4. C. PASINI, Inventario agriografico dei manoscritti greci dell'Ambrosiana, Bruxelles, 2003, $111)$. 
dans Les manuscrits grecs datés de la première moitié du XIV siècle ${ }^{49}$. Le Rapport de Pilate à Tibère, dans le gr. 770, est placé après la Déclaration de Joseph d'Arimathée, f. $20^{\vee}-25$, elle-même précédée des Actes de Pilate (recension A), f. $7-20^{\circ}$. Il se poursuit avec la mention de la réception de la lettre par Tibère et le texte de la Comparution de Pilate, f. $27^{\mathrm{v}}-29$ (suit le récit de la Passio Demetrii). C'est donc tout un cycle de Pilate qui est rassemblé au début de ce manuscrit ${ }^{50}$.

Ce manuscrit a été lu par Fabricius ${ }^{51}$ et par Thilo ${ }^{52}$, qui lui attribue aussi la lettre A. Une note écrite sur un feuillet de papier placé après le dernier folio «indique que l'Anaphora Pilati est la même que celle qui se trouve chez Fabricius et dans les Addenda au Codex Apocryphus. L'auteur de cette note est peut-être Borge (Birgerus) Riisbrigh Thorlacius, qui a copié le manuscrit parisien pour A. Birch ${ }^{53}$ ", ce que confirme Birch ${ }^{54}$, qui ajoute qu'il est sûr que Fabricius a utilisé le même manuscrit. Mais la comparaison du texte édité par Fabricius et de celui édité par Birch met en lumière des divergences communes avec le manuscrit, assez repérables pour que l'on pense que Birch a recopié l'édition de Fabricius plutôt que d'avoir recours directement à la source manuscrite.

B Paris, BNF, gr. $929, \mathrm{XV}^{e}$ s., f. $35-42\left(B H G^{3} 779 \mathrm{yl}\right)$ [Paris. B.N. gr. 939, $\mathrm{XV}^{\mathrm{e}} \mathrm{s}$. , f. 35-42, avec une erreur de numérotation]

Ce manuscrit, auquel la CANT fait à tort référence comme le $\mathrm{gr}$. 939, et qui était autrefois nommé Colbert 5104, est en assez mauvais état. Il s'ouvre sur une succession de plusieurs textes relatifs à Pilate, les mêmes que dans le manuscrit de Paris gr. 770, mais dans un ordre différent: les Actes de Pilate sont suivis du Rapport de Pilate à Tibère et de la Comparution de Pilate, et le cycle se conclut sur la Déclaration de Joseph d'Arimathée, lui-même suivi d'un texte intitulé Anonymi lamentatio de suis peccatis 55 . Le manuscrit se compose de 588 folios et est daté, selon le catalogue de la Bibliothèque Nationale, du XV'c $\mathrm{s}$. Tischendorf l'utilise dans son intégralité, ainsi que Thilo, et tous deux le désignent par la lettre B. Le texte du Rapport de Pilate à Tibère commence par un prologue ressemblant

49. P. GÉHIN et al., Les manuscrits datés de la première moitié du XrV $s$., Paris, 2005, $n^{\circ} 14$, p. $40-43$.

50. H. OMONT, Inventaire sommaire des manuscrits grecs de la Bibliothèque nationale, $1^{\text {ère }}$ partie, p. 135

51. J. A. FABRICluS, Codex Apocryphum Novi Testamenti, p. 456.

52. J. C. Thilo, Codex Apocryphus Novi Testamenti, p. 803.

53. P. GÉHIN et al., Les manuscrits datés de la première moitié du XIve s., p. 42.

54. A. BIRCH, Auctarium Codicis Apocryphi Novi Testamenti Fabriciani, p. XXXI.

55. H. OMONT, Inventaire sommaire des manuscrits grecs de la Bibliothèque nationale, $1^{\text {ere }}$ partie, p. 178.

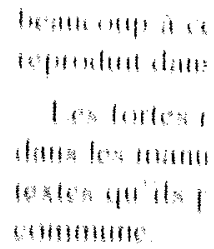

('MUan Mis Mellol Amb

Plichendon! IIU LAOANT de lin Detion la Communuto ablo splst: at

13 Muln, $1 \mathrm{NI}$ Gowl $11 \% 13$

Co menumet lo dểent de fo melenn oots

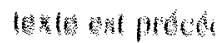
de Pllan (1. ?

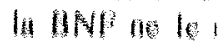
du Nuppart de munuenti do $\mathrm{A}$ pormethe $\mathrm{d}^{\mathrm{sen}}$

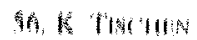

औ) MALTM p. 10
}

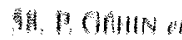

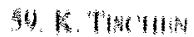

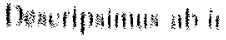

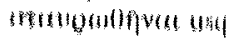
f) $10 \%$

oj 1 Dnytus

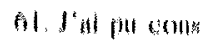

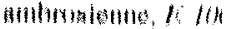




\section{'ERINE BAUDOIN}

iècle ${ }^{49}$. Le Rapport aration de Joseph late (recension $\mathrm{A}$ ), tre par Tibère et le I Passio Demetrii). le ce manuscrit ${ }^{50}$.

ui attribue aussi la ternier folio « indiIve chez Fabricius : note est peut-être scrit parisien pour âr que Fabricius a par Fabricius et de ; avec le manuscrit, lition de Fabricius

N. gr. 939, $\mathrm{XV}^{\mathrm{e}} \mathrm{S}$,

: le $g r .939$, et qui .ll s'ouvre sur une dans le manuscrit late sont suivis du le cycle se conclut d'un texte intitulé pose de 588 folios $X V^{e} \mathrm{~s}$. Tischendorf ignent par la lettre logue ressemblant

s., Paris, $2005, \mathrm{n}^{\circ} 14$

ibliothèque nationale,

., p. 42

i, p. XXXI.

ibliothèque nationale,

\section{ITMOINS MANUSCRITS DU RAPPORT DE PILATE À TIBÈRE}

bouncoup à celui que le gr: 770 place en tête des Acta Pilati, et que Tischendorf veproduit dans son édition à cet endroit-là ${ }^{56}$

Ler fortes ressemblances dans l'environnement du Rapport de Pilate à Tïbère duni log manuscrits de Paris, $g r .770$ et $\mathrm{gr}$. 929, ainsi que la grande proximité des Waksug quls présentent, laissent penser que ces deux manuscrits ont une source gommune.

CHilun, Bibl. ambrosienne, E 100 sup (307), xiI" s., f. 5-6 $\left(B H G^{3} 779 y \mathrm{y}\right)$

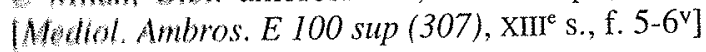

Tínchendorf date ce manuscrit du XII ${ }^{\mathrm{e}} \mathrm{s}$. environ et le reproduit dans son intégraI1t. L. CANT et le catalogue de Milan le datent du XIII $\mathrm{s}$. Notre texte est précédé 14 Oeclaration de Joseph d'Arimathée et suivi sans solution de continuité de la comparution de Pilate, f. $6^{\mathrm{v}}-7^{\mathrm{v}}$, elle-même suivie d'un texte intitulé Dorothei ubb aptst. ad fratrem ${ }^{57}$.

10 Puts, BNF, Coisl. 117, 1333-1334, f. 234-235 (BHG $779 \mathrm{yl}$ ) [Paris. B.N. Colve 11\%, 1332, f. 234-236]

Ce manuscrit comporte 236 folios. Il date de 1333-1334, selon Géhin, qui le decrit de façon exhaustive ${ }^{58}$ (la CANT et Tischendorf indiquent 1332). Son uncienne cote est 64 . Tischendorf en copie une partie ${ }^{59}$. Dans le manuscrit, notre toxle est précédé des «discours d'Isaac le Syrien». Il est suivi de la Comparution de Pilate (f. 235-236), sans solution de continuité, si bien que le catalogue de Ii BNI ne le mentionne pas ${ }^{60}$. Le manuscrit s'achève sur ce texte. La version du Rupport de Pilate à Tibère qu'il propose est très proche de celle livrée par le mamuscrit de Milan E 100 sup (307), pour autant que l'édition de Tischendorf permette d'en juger ${ }^{61}$.

4o. Tischendorf, Evangelia Apocrypha, p. 211.

57. 1. MARTINI-D. BASSI, Catalogus codicum graecorum Bibliothecae Ambrosianae, t. 1, 1. 140.

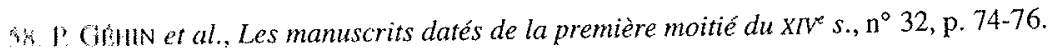

59) K. TISCHENDORF, Evangelia Apocrypha, p. LXXVIII: «D i.e. Coisl. CXVII. a. 1332. scriptus.

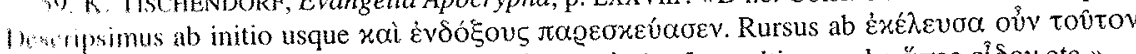

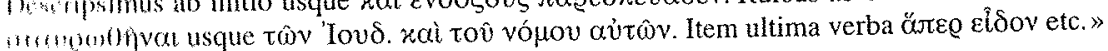

(it) R. DrvneEsse, Catalogue des manuscrits grecs, $2^{e}$ partie: le fonds Coislin, Paris, 1945 , $110 \%$

61. lat pu consulter le manuscrit de Paris, BNF, Coisl. 117, mais pas celui de Milan, Bibl. imblusienne, E 100 sup (307). 


\section{B. Une version brève du Rapport de Pilate à Tibère}

Avant de donner la liste des autres manuscrits, M. Geerard donne les références d'un manuscrit qui n'existe pas, Lincoln. 117, 1333/34, f. 235-236. Il s'agit vraisemblablement d'une confusion avec le manuscrit Paris, BNF, Coisl. 117, 1333-1334, f. 234-235, qui a le même numéro et les mêmes références de folios; mais alors je ne comprends pas la raison du renvoi à ce manuscrit déjà mentionné65. Il fait aussi mention de l'édition de G. F. Abbott, The Report and Death of Pilate, The Journal of Theological Studies 4, 1903, p. 83-86, qui dans $B H G^{\text {na }}$ porte la référence $B H G 779 \mathrm{z}$. On retrouve la même version dans le manuscrit Sinait. gr. $532, \mathrm{XV}^{\mathrm{e}}-\mathrm{XVI} \mathrm{I}^{\mathrm{e}} \mathrm{s}$, f. $44-47^{\mathrm{v}}$, classé par M. Geerard parmi les manuscrits CANT 66 et dans la $B H G^{3}$ sous $B H G 779 \mathrm{z}$, epitome. Cette désignation comme epitome est pertinente pour cette version brève; elle est en revanche inadéquate, comme mentionné plus haut, pour le manuscrit d'Oxford Lincoln 1 , et pour les autres références commençant par $779 \mathrm{z}$ dans la $B H G^{\text {na }}$

La référence du manuscrit consulté par Abbott n'est pas connue, mais on peut être sûr qu'il ne s'agit pas du manuscrit du Sinaï: Abbott précise que le soleil est représenté par un symbole dans son manuscrit ${ }^{66}$ alors que dans le manuscrit du Sinaï ce mot est écrit en toutes lettres; en outre, si certes les deux textes sont très semblables, les variantes sont tout de même nombreuses, comme on peut le voir en les comparant. Ce texte classé par la CANT dans la recension A est peut-être légèrement plus proche des manuscrits du deuxième groupe de la recension $\mathbf{B}$, mais sa brièveté ne permet pas de l'affirmer. J'ai pu consulter l'édition d'Abbott ainsi qu'un manuscrit:

62. K. TISCHENDORF, Evangelia Apocrypha, p. LXXVII, «E i.e. Harleianus 5636, saeculi fere

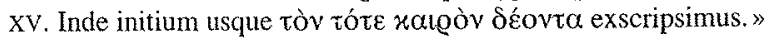

63. Le catalogue groupe ces deux textes sous le même titre.

64. A Catalogue of the Harleian Manuscripts in the British Museum, t. 3, p. 283.

65. Il existe bien un manuscrit de Lincoln College à Oxford avec le Rapport de Pilate à Tibère mais c'est le Lincoln I (cf. CANT 65).

66. G. F. ABBOTT, «The Report and Death of Pilate», The Journal of Theological Studies, 4, 1903, p. 84 : «sign for the sun, with accent and breathing». 

Athen. 2972, $\mathrm{XV}^{\mathrm{e}}-\mathrm{XVI} \mathrm{I}^{\mathrm{e}}$ s., f. $112^{\mathrm{v}}-116^{\mathrm{y}}\left(B H G^{\mathrm{ma}} 779 \mathrm{zc}\right)$

Ce manuscrit est, par sa référence dans la $B H G^{\text {na }}$, lié au manuscrit de Panteleimon 114, et au manuscrit d'Oxford Holkham gr. 9. Cependant je n'ai pas réussi à trouver mention de ce manuscrit dans les catalogues d'Athènes, et J.-M. Olivier ne fait pas mention d'autres catalogues qui pourraient le répertorier. Il n'est pas mentionné non plus dans une description du manuscrit qui ne s'intéresse qu'aux œuvres de Marc Eugène d'Éphèse ${ }^{72}$.

La consultation de la copie de ce manuscrit à l'TRHT enseigne que le Rapport de Pilate à Tibère y est suivi d'un texte qui semble tenir à la fois de la Comparution de Pilate et de la Réponse de Tibère, et qui s'achève sur la mort de Pilate. L'ensemble est ensuite suivi de quatre feuillets blancs avant que ne commence au feuillet 121 un traité sur la foi orthodoxe de Marc d'Éphèse.

Athos, Monastère de Panteleimon, $114, \mathrm{XV}^{\mathrm{e}}$ s., f. 109-116 ${ }^{\mathrm{v}}$ (BHG $\left.{ }^{\mathrm{na}} 779 \mathrm{zc}\right)$ [Athon. Panteleem. 114, XV' s., f. 109]

Selon ce qu' indique le catalogue des bibliothèques du Mont Athos ${ }^{73}$, le Rapport de Pilate à Tibère est précédé dans ce manuscrit de la vie de saint Jean Calybite et suivi de la vie de saint Alexis. Ces deux hagiographies sont assez proches; on peut se demander ce qui a pu pousser un copiste à insérer entre elles le Rapport de Pilate à Tibère.

Athos, Monastère de Vatopedi, 37, $\mathrm{XV}^{\mathrm{c}}$ s., f. $1-8^{\mathrm{v}}$ (BHG $\left.\mathrm{\text {na }} 779 \mathrm{zd}\right)$ [Athon. Batop. 37]

Le Rapport de Pilate à Tibère ouvre ce manuscrit. Il est suivi d'un texte intitulé

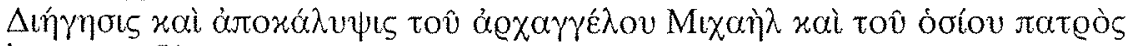

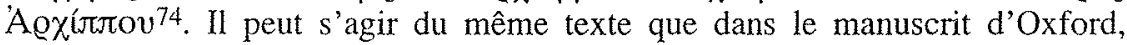
Holkham gr. 9, la vie d'Archippos, fêté le 6 septembre; on pourrait faire l'hypothèse de la proximité des textes du Rapport de Pilate à Tibère livrés respectivement par le manuscrit d'Oxford et par celui de Vatopédi; il faudrait alors vérifier si la distinction entre la référence $B H G 779 \mathrm{zc}$ (manuscrit d'Oxford Holkham gr. 9) et $B H G 779 z d$ (ce manuscrit) doit être maintenue, ou si les deux manuscrits doivent être regroupés sous une même référence, selon le degré de proximité des autres manuscrits classés sous les cotes $B H G 779 \mathrm{zc}$ et $B H G 779 \mathrm{zd}$.

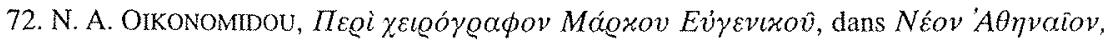

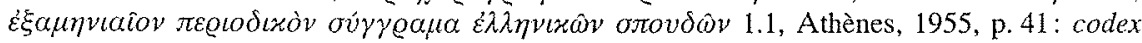
ath. 2972.

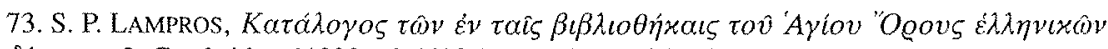

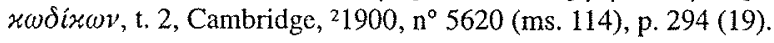

74. S. Eustratiades-ARCADIos VATOPEdINos, Catalogue of the Greek Manuscripts in the Library of the Monastery of Vatopedi on Mount Athos, Cambridge, Mass., 1924, p. 13. 
Munich. Bibl. de Bavière, gr. 524, XIV s., f. 122-124 (BHG na 779zd) [Monac. yr. 524, XIV"s., f. 122-124]

Le catalogue de Munich indique la présence du Rapport de Pilate à Tibère Junis of manuscrit, ou il est clos par une doxologie propre ${ }^{75}$. Il est suivi d'un

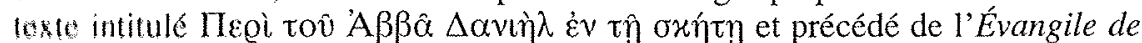
Niondeme ${ }^{76}$, lui-même précédé d'une homélie d'Épiphane sur le Saint Sépulcre, Jameph d'Arimathée, Nicodème, et la descente aux Enfers ${ }^{77}$.

Huncuce, Bibl. laurentienne, VIII. 18, XIV s, f. 218v-221 [Comparution de Pilate comprise] (BHG 779zd) [Flor. Laurent. VIII. 18 ]

Duns ce manuscrit, le Rapport de Pilate à Tibère est suivi de la Comparution d Plate, ce qui n'est pas indiqué par le catalogue mais révélé par la citation de

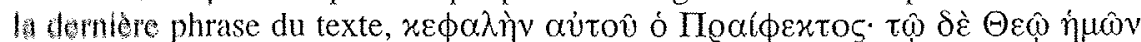

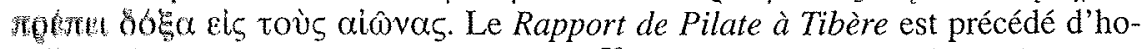
núlies: d'Abba loannis, cénobite du Sinaï ${ }^{78}$. Ce manuscrit est référencé comme $0110779 \%$ d, c'est-à-dire avec le même sigle que le manuscrit de Vatopédi 37 et le munuscrit de Munich gr. 524.

Alhonew, BN, 352, XV $-\mathrm{XVI}^{\mathrm{e}}$ s., f. $172^{\mathrm{v}}-180\left(B H G^{\text {na }} 779 \mathrm{ze}\right)\left[\right.$ Athen. 352, XV $\mathrm{XV}^{\mathrm{e}}-\mathrm{XVI}^{\mathrm{e}}$ S., (. $172 \% 1801$

Duns ce manuscrit, le Rapport de Pilate à Tỉbère est précédé de l'Évangile de

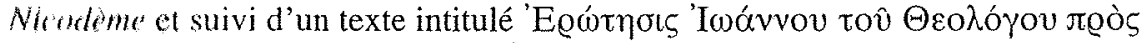

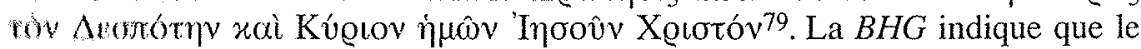
liapport de Pilate à Tibère est lié dans ce manuscrit à la Comparution de Pilate.

t suivi d'un texte intitulé

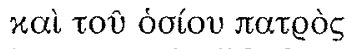
le manuscrit d'Oxford, on pourrait faire l'hypòre livrés respectivement udrait alors vérifier si la ixford Holkham gr: 9) et deux manuscrits doivent de proximité des autres zd.

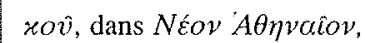
Athènes, 1955 , p. 41 : codex

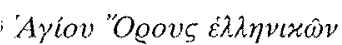

e Greek Manuscripts in the tass., 1924 , p. 13

95. I HARDT. Catalogus codicum manuscriptorum graecorum Bibliothecae Regiae Bavaricae, Muinh, 1812,1,5, p. 293.

76. 1. ciltalogue en donne le titre, vлó

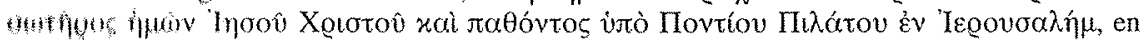
U1:a!n 4u' il n'est pas repris par Fabricius. Une note manuscrite en marge, dans l'édition que nous sum: contultec (sur www.digitale-sammlungen.de), corrige avec indignation cette assertion: Est fiwmethin Nicodemi!

7. 1. alutogute précise que ce texte se trouve mutatis mutandis chez Fabricius, et qu'il est ristumb lans le catalogue de Florence cité ci-dessous (Florence, Bibl. laurentienne, viII. 18), et Iman ietui de Venise (il s'agit alors du manuscrit de la bibliothèque marcienne 11,42 , qui contient

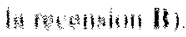

7h. A M BANDINI, Catalogus codicum manuscriptorum Bibliothecae Medicae Laurentianae, 1. 11:rence. 1764. p. $362(6)$.

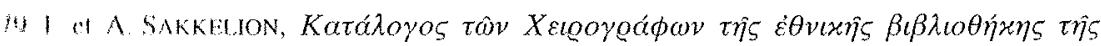
thisituk. P. 59) (8). 


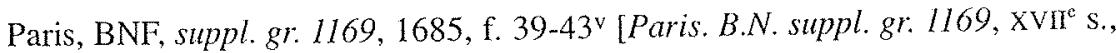
f. $\left.39-43^{v}\right]$

Le catalogue de la BNF indique la date de ce manuscrit, 1685, quand la CANT donne simplement XVII ${ }^{e}$ s. Le manuscrit se compose de 48 folios, s'ouvre sur un «fragment d'exégèse par demandes et réponses», sur le premier folio, et se poursuit avec quelques textes relatifs au cycle de Pilate, l'Evangile de Nicodème, le Rapport de Pilate à Tibère (f. 39-43v) et, sans solution de continuité, la Réponse

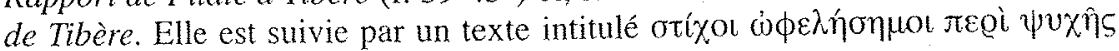

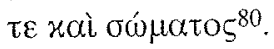

Le texte reproduit est «une version délayée de l'Anaphora Pilati telle que l'a éditée Tischendorf ${ }^{81} \gg$. Le catalogue de la BNF note en outre que le texte reproduit «est assez voisin de celui du Manuscrit $A »$, c'est-à-dire le manuscrit de Paris, gr. 770,1315 , f. $25-27^{*}$, et que la Réponse de Tibère qui le suit est aussi une «version délayée». La lecture du texte permet de remarquer qu'il est issu de la

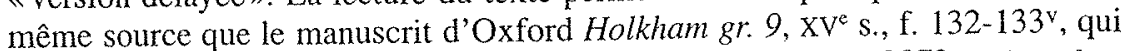
est référencé comme $B H G 779 \mathrm{zc}$ et que le manuscrit d'Athènes $2972, \mathrm{XV}^{\mathrm{e}}-\mathrm{XVI}^{\mathrm{c}} \mathrm{s}$, f. $112^{v}-116^{v}$, qui a la même référence dans la $B H G$. Il pourrait donc être pertinent de classer ce manuscrit en $B H G 779 \mathrm{zc}$. Permettre ce rapprochement semble être le principal intérêt de ce manuscrit qui est par ailleurs assez tardif et corrompu.

Athènes, BN, 2187, XV s, f. 206-207 [Athen. 2187, XV'e., f. 206-207]

Il est fait mention dans la CANT d'un manuscrit d'Athènes, $2187, \mathrm{XV}^{\mathrm{e}} \mathrm{s}$, f. 206207: ce manuscrit contient à partir du folio 193 l'Évangile de Nicodème l2 $^{82}$ et le

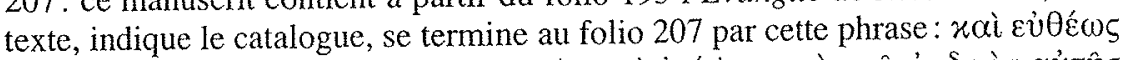

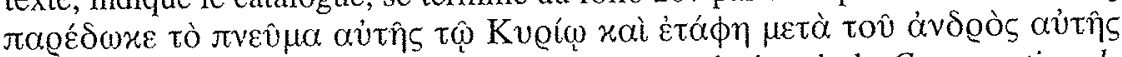

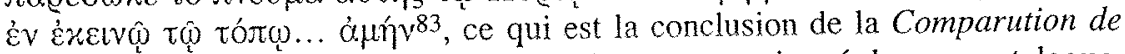
Pilate $^{84}$. Le Rapport de Pilate à Tibère n'est pas mentionné dans ce catalogue, mais peut très bien figurer dans le manuscrit juste avant la Comparution de Pilate.

Enfin, M. Geerard cite un manuscrit du Vatican, Vat. gr: $417, \mathrm{XI}^{\mathrm{c}}$ s., f. 366 , qui contient en fait le texte de la recension $\mathrm{B}$ du Rapport de Pilate à Tibère ainsi que la Comparution de Pilate - c'est la raison pour laquelle il est doublement

80. C. Astruc-M.-L. CONCASTI, Catalogue des manuscrits grecs, $3^{c}$ partie: le supplément grec, $n^{\circ}$ 901-1371, Paris, 1960, p. 334-335.

81. Ibid., p. 334 (3).

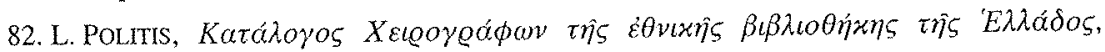
Q̉. 1857-2500, p. 217, 2187 (54).

83. «Et aussitôt, elle rendit l'esprit au Seigneur, et fut enterrée avec son mari en ce lieu... amen. » Il s'agit de Procla, l'épouse de Pilate. Cf. Comparution de Pilate, R. Gounelle (tr.), dans Écrits apocryphes chrétiens, t. 2, p. 322-327.

84. K. TISCHENDORF, Evangelia Apocrypha, p. 455. 
gr: $1169, \mathrm{XVIr}^{\mathrm{e}} \mathrm{s}$.

5, quand la CANT os, s'ouvre sur un $r$ folio, et se pour- de Nicodème, le nuité, la Réponse

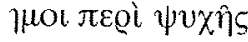

'ilati telle que l'a le texte reproduit inuscrit de Paris, uit est aussi une a'il est issu de la f. $132-133^{\circ}$, qui ?972, XV'-XVI $\mathrm{e}$., mo être pertinent nent semble être if et corrompu.

\section{;-207]}

$87, X^{\mathrm{e}}$ s, f. 206 Iicodème ${ }^{82}$, et le

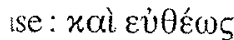

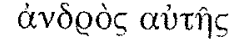
Somparution de as ce catalogue, comparution de

; XII s., f. 366 , 'e à Tibère ainsi est doublement

tie: le supplément

ऽ $\tau \hat{\eta} \varsigma ~ E \lambda \lambda \alpha \dot{\alpha} \delta \varsigma$,

mari en ce lieu... IUNELLE (tr.), dans

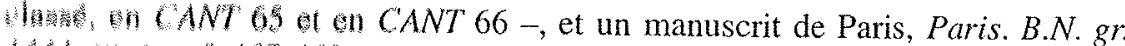

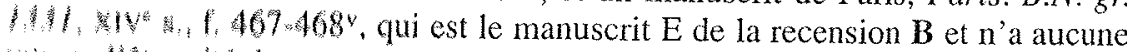

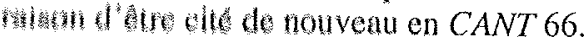

\section{Nouveaux témoins de la recension A (CANT 66)}

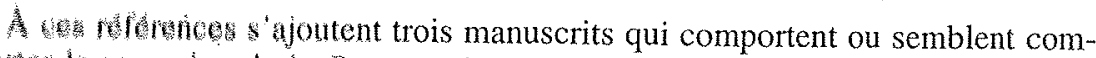

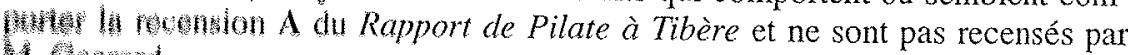
1. Tisard

Whin, Mibl mbrosienne, $C 92$ sup. (N 155; N 158), XIV s. (2/4), f. 327-328 (hilly

Co munuatent contient une version du Rapport de Pilate à Tibère à laquelle I0 sululogue attribue à juste titre la référence $B H G 779 y{ }^{85}$. Elle est suivie,

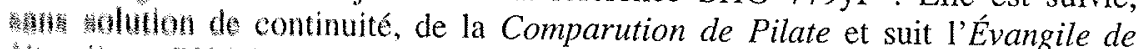
Whathe (1110779t), lui-même précédé d'un texte intitulé Apocalypsis Methodii W10 20130). Sa lecture révèle qu'il est la copie exacte du manuscrit de Londres himlan 10

Jumbulem, Monastere de Saint-Sabas, 418 , début XIV ${ }^{\mathrm{e}}$ S., f. $226^{\mathrm{V}}$-fin

(In poui supposer, grâce à la succincte mention faite dans la notice du manus-

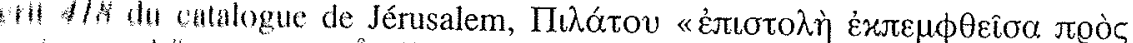

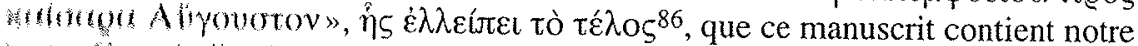
16 Glle indlication ressemble légèrement plus à l'ouverture des manuscrits de lin monmon A, mais sa brièveté ne me permet pas de trancher. Le texte est précédé I'in discours de lean Chrysostome «Sur le fils de la veuve».

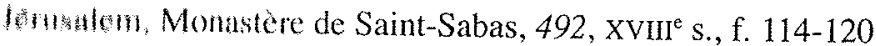

Imis lu description du manuscrit 492 proposée par le catalogue de Jérusalem, on

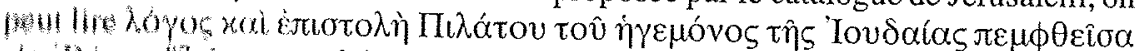

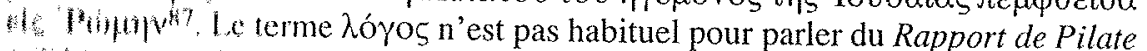
Hithin main la fin de la description est très proche des titres des manuscrits de

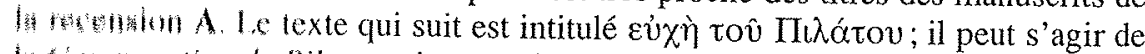

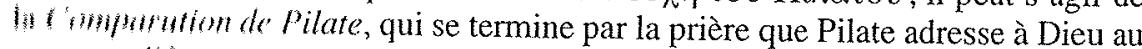
mintian d'cltre cxécuté par le préfet sur ordre de Tibère. Le dernier paragraphe de

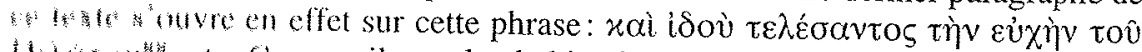

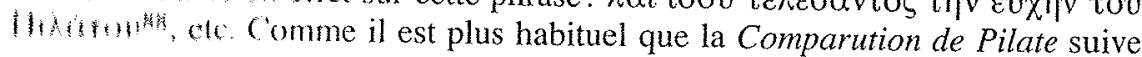

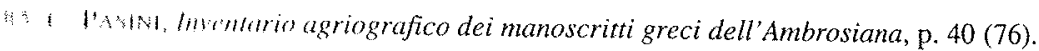

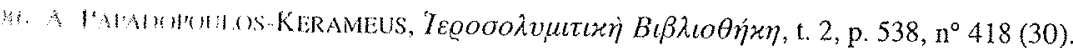

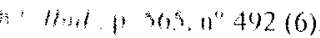

1. 11:411 Nimet, Evangelia Apocrypha, Paradosis Pilati 10, p. 455.
} 
les textes de la recension $\mathbf{A}$, on peut faire l'hypothèse que le manuscrit 492 en contient une copie. Le Rapport de Pilate à Tibère est précédé d'un texte sur la vie

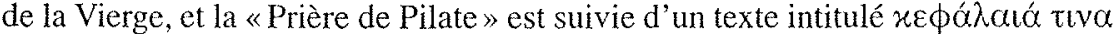
tố óoíou Níxwvos.

Ainsi de nouveaux témoins du Rapport de Pilate à Tibère apparaissent-ils pour éclairer notre lecture du texte et notre connaissance des liens entre les manuscrits. La $C A N T$ donne les références de quatorze témoins de la recension $\mathbf{B}$ (CANT 65 ) et de vingt témoins de la recension A (CANT 66). Parmi les témoins de la recension $\mathbf{B}$, cinq sont les manuscrits édités par Tischendorf:

- Vienne, BN, theol.gr, $247,2^{\circ}$ moitié du XV $\mathrm{V}^{\mathrm{e}} \mathrm{s}^{89}$, f. $148^{\mathrm{v}}-151$

- Turin, c. II, 5 (Pasini gr. 302), XII' ${ }^{\mathrm{e}}$ S., f. $60^{\mathrm{v}}$

- Venise, Bibl. marcienne, $I I, 42, X^{2} \mathrm{e}^{\mathrm{e}}-\mathrm{XIV}^{\mathrm{e}} \mathrm{s}$., f. $130^{\mathrm{V}}-132$

- Paris, BNF, gr. 1019 A, XIV s., f. $236^{\mathrm{V}}-237^{\mathrm{V}}$

- Paris, BNF, gr. 1331, XIV s. f. f. 467-468

trois ne contiennent pas le texte de la recension $\mathbf{B}$ du Rapport de Pilate à Tibère, trois ont été classés par erreur dans la recension $\mathbf{A}$ (CANT 66):

- Patmos, Monastère de saint Jean le Théologien, 448, XV s., f. 61-63

- Londres, BL, Add. 25881, XVI ${ }^{\mathrm{e}}$ s., f. 255-257v

- L'Escurial, Bibl. royale, $\omega$ IV. 18 (570), XV s., f. 103 ${ }^{\mathrm{v}}-105$;

et six contiennent bien un texte inédit de la recension $B$ :

- Athos, Grande Laure, K. 64, 1634, f. 91-95v

- Vatican, Bibl. vaticane, $g r$. 417, XII s., f. $365^{\mathrm{r}}-366$

- Londres, BL, Harley 5556, 1714, f. 29-30v

- Paris, BNF, suppl. gr. 78, XVII' s., f. 200-202

- Athènes, BN, autrefois Métochion du Saint-Sépulcre 303, XIV-XV's., f. $158-159^{\circ}$

- Milan, Bibl, ambrosienne, H 22 sup. (426), XVe s., f. 7-7v.

J'ajoute aux quatorze manuscrits désormais correctement répertoriés sept témoins, portant leur nombre à vingt-et-un:

- Londres, BL, Burney 342, 1709, f. 91-94

- Jérusalem, Patriarcat, Saint-Sépulcre $238, \mathrm{XVIII}^{\mathrm{e}}$ s., f. $62^{\mathrm{v}}-64^{\mathrm{v}}$

- Athos, Grande Laure, M. 68, 1772, f. 208-209

- Athènes, BN, 432 [130], XVI $\mathrm{s}$.

- Athènes, BN, 2069, XVIII ${ }^{\mathrm{C}} \mathrm{XIX}^{\mathrm{e}}$ s., f. 96-104

- Oxford, Bibl. bodléienne, Lincoln $1,2^{\mathrm{e}}$ quart du $X \mathrm{~V}^{\mathrm{e}}$ s., f. $4^{\mathrm{v}}-5^{\mathrm{v}}$

- Milan, Bibl. ambrosienne, A 79 sup. (T 223, T 219, T 224), 1517, ff. $1-2^{y}$

89. H. HUNGER-W. LACKNER-C. HANNICK, Katalog der griechischen Handschriften der österreichischen Nationalbibliothek, 3/3: Codices theologici 201-337, Vienne, 1992. 
Parmi les témoins de la recension $\mathbf{A}$, outre les cinq manuscrits de Tischendorf:

- Paris, BNF, $g r .770,1315$, f. $25-27^{\mathrm{v}}$

- Paris, BNF, $g r$ : $929, \mathrm{XV}^{\mathrm{e}}$ s., f. $35-42$

- Milan, Bibl. ambrosienne, E 100 sup (307), XII" s., f. 5-6r

- Paris, BNF, Coisl. 117, 1333-1334, f. 234-235

- Londres, BL, Harl. 5636, XIV s. f. 26-30,

Gคต 1 ig pour ingmusthts (ii) $1 \mathrm{ICN}$ anoun do la

ato

$1: 1 \% 03$

onters sept

1). 1517 si on retire les trois manuscrits improprement classés, le manuscrit du Sinaï qui propose une version brève, le manuscrit de Paris $g r .1331$ mentionné aussi en CANT 65 - à raison - et le manuscrit du Vatican $g r .417$ qui contient la recension B suivie de la Comparution de Pilate, on voit que neuf manuscrits contiennent un texte inédit de la recension $\mathrm{A}$ :

- Oxford, Bibl. bodléienne, Holkham gr. $9, \mathrm{XV}^{\mathrm{e}}$ s., f. 132-133v

- Athen. 2972, XV'-XVI ${ }^{\mathrm{e}}$ S., f. $112^{\mathrm{v}}-116^{\mathrm{v}}$

- Athos, Monastère de Panteleimon, $114, \mathrm{XV}^{\mathrm{e}}$ s., f. 109-116

- Athos, Monastère de Vatopedi, $37, X^{e}$ s., f. 1-8v

- Munich, Bibl. de Bavière, gr. 524, XIV s., f. 122-124

- Florence, Bibl. laurentienne, VIII. 18, XIV e s, f. $218^{\mathrm{v}}-221$ [Comparution de Pilate comprise]

- Athènes, BN, 352, XV'-XVI' s., f. $172^{\mathrm{v}}-180$

- Paris, BNF, suppl. gr. 1169, 1685, f. 39-43

- Athènes, BN, 2187, XV ${ }^{\mathrm{e}}$ s, f. 206-207;

aux quatorze manuscrits désormais correctement répertoriés, j'ajoute trois témoins, portant leur nombre à dix-sept :

- Milan, Bibl. ambrosienne, C 92 sup. $(N$ 155; $N$ 158), XIV s. (2/4), f. $327-328$

- Jérusalem, Monastère de Saint-Sabas, 418 , début XIVe s., f. $226^{\vee}$-fin

- Jérusalem, Monastère de Saint-Sabas, 492, XVIII ${ }^{\mathrm{e}}$ s., f. 114-120.

Ces nombres sont appelés à être modifiés, et la liste présentée ici n'est sans doute pas exhaustive. Des lectures et des rapprochements que permettent ces manuscrits, je propose de retenir surtout la distinction à effectuer entre deux groupes de manuscrits proposant la recension $\mathbf{B}$, et l'existence d'une version brève du texte, sommaire ou résumé du Rapport de Pilate à Tibère. Mais c'est à Mademoiselle Gabriella Aragione, de la Faculté autonome de Théologie protestante de l'Université de Genève, que revient la tâche, dans le cadre d'un projet de recherche soutenu par le F.N.R.S., de préparer une collation et une édition critique des manuscrits dans le cadre d'un projet éditorial de l'Association pour l'Étude de la Littérature Apocryphe Chrétienne, pour publication dans le Corpus christianorum, Series apocryphorum. 
RÉSUMÉ: Certaines références des témoins manuscrits du Rapport de Pilate à Tibère dans la Clavis Apocryphorum Novi Testamenti sont parfois imprécises, et la liste est apparue incomplète. La consultation des catalogues de manuscrits des bibliothèques a permis de faire apparaître dix nouveaux témoins de ce texte apocryphe connu par deux recensions, A (CANT 66) et $\mathbf{B}$ (CANT 65); une première collation montre, dans la recension $\mathbf{B}$, l'existence de deux familles de manuscrits qui proposent notamment un ordre narratif différent, et permet de proposer des hypothèses de liens entre les manuscrits; enfin, un nouveau témoin d'une version brève du Rapport de Pilate à Tibère est apparu.

ABSTRACT: Some of the references of the Report of Pilate to Tiberius in the Clavis Apocryphorum Novi Testamenti may lack of precision and the list appears to be uncomplete. Consulting the libraries catalogs lead to the discovery of ten new witnesses of this apocryphal text known by two recensions called A (CANT 66) and B (CANT 65); a first collation reveals the existence in the $\mathbf{B}$ recension of two families of manuscripts that offer in particular a different narrative order, which allows to form hypotheses about the links existing between the manuscripts; and a new witness of a short version of the Report of Pilate was brought to light. 